\title{
THE FEBRUARY MEETING IN CINCINNATI
}

The four hundred sixty-fifth meeting of the American Mathematical Society was held at the University of Cincinnati on Friday and Saturday February 23-24, 1951. There were about 85 registrations including the following $\mathbf{5 7}$ members of the Society:

J. E. Adney, E. S. Allen, Emilio Baiada, I. A. Barnett, W. R. Baum, L. G. Belai, W. D. Berg, Dewey Blair, Louis Brand, R. W. Bryant, E. D. Cashwell, Lamberto Cesari, V. F. Cowling, E. H. Crisler, R. E. Fullerton, Franklin Haimo, O. G. Harrold, Hugo Heermann, Carl Holtom, W. E. Jenner, H. K. Justice, Frank Levin, D. J. Lewis, H. D. Lipsich, Lee Lorch, C. I. Lubin, R. W. MacDowell, H. B. Mann, William Marcaccio, Gaylord Merriman, E. J. Mickle, H. L. Miller, C. N. Moore, W. B. Morgan, Tadasi Nakayama, Zeev Nehari, H. S. Pollard, O. W. Rechard, P. V. Reichelderfer, Haim Reingold, M. E. Rice, R. A. Roberts, C. H. Rust, H. J. Ryser, W. C. Sangren, K. C. Schraut, M. E. Shanks, Edward Silverman, T. F. Smith, W. S. Snyder, V. C. Stechschulte, E. G. Swafford, Otto Szász, E. F. Trombley, J. A. Ward, E. F. White, J. W. T. Youngs.

The Committee to Select Hour Speakers for Western Sectional Meetings had issued two invitations, one to Professor Zeev Nehari of Washington University and the second to Professor Tadasi Nakayama of Nagoya University and the University of Illinois. Professor Nehari spoke on Bounded analytic functions at 2:00 P.M. Friday while Professor Nakayama addressed the Society on Galois theory of rings at 10:30 A.M. Saturday.

Presiding officers for the various sessions were Professors Otto Szász, W. S. Snyder, E. S. Allen, and I. A. Barnett.

The University of Cincinnati entertained the Society at a tea on Friday afternoon and there was a dinner on Friday evening at the Sinton Hotel. Dean Greene welcomed the guests on behalf of the University of Cincinnati and Professor Shanks responded. The dinner was characterized by an air of friendly informality, possible perhaps only at one of the regional meetings.

The list of papers presented follows. Those abstracts indicated by the letter " $t$ " were presented by title. Professor Koenig was introduced by Professor J. W. T. Youngs and Professor Fortet by Professor G. E. Forsythe.

\section{Algebra AND Theory of Numbers}

\section{6t. A. A. Albert: On simple alternative rings.}

The purpose of this note is that of proving the following result. Let $C$ be a simple alternative ring which contains an idempotent not its unity quantity. Then $C$ is either 
associative or is a Cayley algebra over its center. The result is proved by a procedure similar to that used in recent work on simple power-associative commutative rings and no restriction on the characteristic of $C$ is required. (Received January 15,1951.)

\section{7t. Bjarni Jónsson: An infinite Boolean algebra without proper} automorphisms.

A simply ordered set $C$ is constructed which has the following properties: (i) Each member of $C$ has a character of the form $\left(\omega_{\alpha}, \omega_{0}^{*}\right)$ for some $\alpha>0$. (ii) No two members of $C$ have the same character. (iii) Each gap of $C$ has a character of the form $\left(\omega_{\alpha}, \omega_{0}^{*}\right)$ for some $\alpha \geqq 0$. (iv) $C$ is cofinal with $\omega_{0}$ and coinitial with $\omega_{0}^{*}$. Replacing each member of $C$ by an ordered pair of elements and completing the resulting set by cuts, one obtains a simply ordered set $S$ whose interval topology is compact and zero-dimensional and has no proper homeomorphisms onto itself. Hence the Boolean algebra of open and closed subsets of $S$ has no proper automorphisms. This solves Problem 74 in G. Birkhoff, Lattice theory (rev. ed.). (Received January 10, 1951.)

\section{ANALYsis}

\section{Lamberto Cesari: Eilenberg's inequality for Lebesgue area.}

In 1943 S. Eilenberg and O. Harrold proved a general form of inequality involving the $\alpha$-dimensional measures of a set $H$ in a general space. Eilenberg, L. C. Young, and the author have shown that in a particular case, involving the elementary area of a polyhedral surface in $E_{3}$ and the lengths of elementary curves, a simple formulation and proof are possible. The author has also applied such a case to the calculus of variations with the aim of extending to parametric continuous surfaces an existence theory based only upon geometrical considerations and the concept of lower semicontinuity. The author has proved the following independent formulation of the statement of Eilenberg and Harrold, involving the concepts of Lebesgue area and of ordinary length: Given a parametric continuous surface $S: x=x(w), w \in Q[w=(u, v)$, $x=\left(x^{1}, x^{2}, x^{3}\right), Q$ unit square $]$ and a real one-valued function $f(x), x \in E_{3}$, such that $\left|f(x)-f\left(x^{\prime}\right)\right| \leqq\left|x-x^{\prime}\right|$, let $t_{0}=\max f[x(w)], w \in Q^{*}, t_{1}=\max f[x(w)]$, w $\in Q$, and suppose $t_{0}<t_{1}$. For any $t_{0}<t<t_{1}$ let $A(t)$ be the maximal connected open set $A(t) \subset Q$ containing $Q^{*}$ and such that $f[x(w)]<t$. The part of the boundary $A^{*}(t)$, besides $Q^{*}$, has an image $m(t)$ on $S$. By extension of the ordinary length, calling $l(t)$ the "length" of $m(t)$, the following inequality holds: $L(S) \geqq \int_{t_{0}}^{t} l(t) d t$, where $L(S)$ is the Lebesgue area of $S$. (Received November 29, 1950.)

\section{J. F. Koenig: Regions of the zeros of polynomials with real or} complex coefficients.

Using the Bilharz-Frank theorem, it can be determined whether or not all zeros of a polynomial with complex coefficients lie within the half-plane to the left of the imaginary axis. Several generalizations of the Bilharz-Frank theorem are given. With these generalizations it can be determined whether or not the zeros of polynomials with real or complex coefficients lie within regions such as: Sectors and sub-sectors of the complex plane; any rectangular infinite strip symmetric about the real axis; region where the real part of $Z$ is less than $-\alpha$ ( $\alpha$ real). When the region of the zeros of a polynomial with real coefficients is to be determined, the real polynomial is trans- 
formed into a polynomial with complex coefficients. The Bilharz-Frank theorem is then applied to the transformed polynomial. Various linear transformations can be used. When the region of the zeros of a polynomial with complex coefficients is to be determined, the complex polynomial is transformed into another complex polynomial. The Bilharz-Frank theorem is then applied to the transformed polynomial. Various linear transformations can be used. These generalizations also give the conditions on the polynomial coefficients such that all zeros will lie within a prescribed region. (Received December 26, 1950.)

\section{H. B. Mann: Some theorems on difference sets.}

A set of different residues $a_{1}, \cdots, a_{k} \bmod v$ is called a difference set $(v, k, \lambda)$ $(v>k>\lambda) \bmod v$ if the congruence $a_{i}-a_{j} \equiv r(v)$ has exactly $\lambda$ solutions for every $r \neq 0$. A number $t$ is called a multiplier of $(v, k, \lambda)$ if $\left\{t a_{i}\right\}=\left\{a_{j}+s\right\}$ for some $s$. The following theorems are proved. (1) If a multiplier $t$ is of even order with respect to a prime divisor $g$ of $v$, then $n=k-\lambda$ is a square if $(t / g)=-1$. If $(t / g)=+1$, then $n$ is either a square or of the form $a^{2} q^{3}$. If moreover $g=v$, then $k=v-1$. We further put $\theta(x)$ $=\sum_{1}^{k} x^{a} i, T(x)=\sum_{0}^{v-1} x^{r}$. Then (2) a prime $p$ is multiplier of $(v, k, \lambda)$ if and only if $\theta(x)^{p} \equiv x^{s} \theta(x)\left(p, x^{v}-1\right)$ for some $s$. If moreover $n \neq 0(p)$, then $\theta(x)^{p-1} \equiv x^{s}\left(p, x^{v}-1\right)$ if $k \neq 0(p)$ and $\theta(x)^{p-1} \equiv x^{s}-T(x)\left(p, x^{v}-1\right)$ if $k \equiv 0(p)$. Both theorems have several corollaries. Theorem 1 implies for instance that $n$ is a square if for some divisor $p$ of $n$ we have $p>\lambda$ and $\left((-1)^{(\nu-1 / 2)} \lambda / p\right)=-1$. Moreover if $p>\lambda,\left((-1)^{(\nu-1 / 2)} \lambda / p\right)=-1$, and $v$ is a prime, then $(v, k, \lambda)$ is impossible. Theorem 2 implies: If 2 is a multiplier of $(v, k, \lambda)$, then 2 divides $n$. If 3 is a multiplier $\bmod (v, k, 1)$ then 3 divides $n=k-1$. (Received December 22, 1950.)

\section{M. E. Shanks: On the existence of measures.}

Let $\phi$ be a finite measure on a set $X$. Let $F$ be the set of all real functions on $X$ and let $\mathcal{F}$ be the set of equivalence classes of $F$ under $\phi$. Then (see A. Rosenthal and H. Hahn, Set functions, University of New Mexico Press, 1948, p. 137) the asymptotic $\phi$-metric makes $\mathcal{f}$ a topological group. In this paper the following converse to the above theorem is proved. Suppose: (1) $\rho\left(f_{1}, f_{2}\right)$ is a quasi-metric on $F$, and $\mathcal{F}$ the set of equivalence classes under $\rho$. (2) With metric $\rho, \mathcal{F}$ is a topological group. $(3) \rho(f, 0)=0$ implies $\rho(|f|, 0)=0$. (4) $0 \leqq g_{n} \leqq f_{n}$ and $\rho\left(f_{n}, 0\right) \rightarrow 0$ imply $\rho\left(g_{n}, 0\right) \rightarrow 0$. (5) If $f_{n}$ is a Cauchy sequence in $\mathcal{f}$ and $f_{n} \rightarrow f$ pointwise, then $\rho\left(f_{n}, f\right) \rightarrow 0$. Then there exists an invariant metric $\rho^{*}$ on $\mathcal{f}$ such that if we define $\phi(A)=\rho^{*}\left(f_{A}, 0\right)$, where $f_{A}$ is the characteristic function on $A, \phi$ is a finite measure on $X$ and the asymptotic metric gives the same equivalence classes in $F$ as does $p$ and the same topology to $\mathcal{F}$. (Received January 11, 1951.)

\section{Otto Szász: On the Gibbs' phenomenon for a class of linear trans-} forms.

Given a function $\phi(t)$ such that $\phi(+0)=1$ and $\int_{0}^{\infty}|d \phi(t)|$ is finite. The series $\phi(h)=\sum_{1}^{\infty} a_{n} \phi(n h)$ is then a regular transform of the series $\sum a_{n}$. For $a_{n}=(\sin n t) / n$ write $\phi(h)=T(h, t)$; the constant $G=\lim \sup (2 / \pi) T(h, t)$, when $h$ and $t$ tend to zero, is called the Gibbs' ratio of the transform. The transform presents a Gibbs' phenomenon if $G>1$. The main result of this paper is that $G=\max _{\zeta>0}(2 / \pi) \int_{0}^{\infty} \phi(t)((\sin \zeta t) / t) d t$. A related result is due to Kuttner. Some particular summability methods and the cor- 
responding Gibbs' phenomenon are then discussed. (Received January 5, 1951.)

\section{Applied Mathematics}

\section{Louis Brand. The algebra of motors.}

The author has shown that a motor $M$ can be regarded as a dual multiple of a unit line vector (vector and tensor analysis): $\boldsymbol{M}=\boldsymbol{m}+\epsilon \boldsymbol{m}_{0}=\left(\lambda+\epsilon \lambda^{\prime}\right)\left(a+\epsilon \boldsymbol{a}_{0}\right)$, where $\lambda, \lambda^{\prime}$ are real scalars, $a, a_{0}$ vectors such that $a \cdot a=1, a \cdot a_{0}=0$ and $\epsilon$ a unit such that $\epsilon^{2}=0$. In the algebra of motors, regarded as dual vectors, the scalar and motor product of two motors are defined as $\boldsymbol{M} \cdot \boldsymbol{N}=\boldsymbol{m} \cdot \boldsymbol{n}+\boldsymbol{\epsilon}\left(\boldsymbol{m} \cdot \boldsymbol{n}_{0}+\boldsymbol{m}_{0} \cdot n\right)$ and $\boldsymbol{M} \times \boldsymbol{N}=\boldsymbol{m} \times \boldsymbol{n}$ $+\epsilon\left(m \times n_{0} \nmid m_{0} \times n\right)$; the former definition differs from the scalar product of von Mises (Zeitschrift für Angewandte Mathematik und Mechanic vol. 4, p. 163), namely $\boldsymbol{M} \cdot \boldsymbol{N}=\boldsymbol{m} \cdot \boldsymbol{n}_{0}+\boldsymbol{m}_{0} \cdot \boldsymbol{n}$. In the algebra of dual vectors thus obtained, all the rules of ordinary vector algebra are still valid. Any three motors $\boldsymbol{M}_{1}, \boldsymbol{M}_{2}, \boldsymbol{M}_{\mathbf{3}}$ for which $m_{1} \cdot m_{2} \times m_{3} \neq 0$ have a unique reciprocal set $M^{1}, M^{2}, M^{3}$, where $M_{i} \cdot M^{j}=\delta_{i}^{i}$. The axes of two reciprocal sets afford a constructive proof of the Theorem of Peterson and Morley. Motor dyadics can be introduced; and the unit motor dyadic is the idemfactor of Gibbs, which can be expressed in the form $M_{1} M^{1}+M_{2} M^{2}+M_{3} M^{3}$. Motor dyadics of this type can be reduced to the form $\Psi+\epsilon \Psi_{0}$, where $\Psi$ and $\Psi_{0}$ are ordinary dyadics and $\Psi_{P}=\Psi_{0}+P O^{-} \times \Psi-\Psi \times P O^{-}$. If $\Psi$ is complete, the motor dyadic $\Psi+\epsilon \Psi_{0}$ has the unique reciprocal $\Psi^{-1}-\epsilon \Psi^{-1} \cdot \Psi_{0} \cdot \Psi^{-1}$. (Received January 10,1951.)

\section{GEOMETRY}

194t. D. O. Ellis: Notes on abstract distance geometry. II. Implications of basality in generalized semimetric spaces.

With the exception of the term basality the present note is couched in the terminology of the foundational paper (David Ellis, Geometry in abstract distance spaces, Publicationes Mathematicae (Debrecen) vol. 2 (1951)). A distance space is called basal if every point of the space forms a complete metric base for the space. In this note it is shown that any basal generalized semimetric space has the property of free mobility and is (1) metrically irreducible, (2) distancially irreducible, (3) irreducible over the ground set. An example of a basal generalized semimetric space is the autometrized Boolean algebra (cf. David Ellis, Autometrized Boolean algebras, I, to appear in the Canadian Journal of Mathematics). (Received November 30,1950.)

\section{5t. Ira Rosenbaum: The theory of the congruence of point pairs.}

By Veronese and R. L. Moore the theory of geometric congruence is based on that of the congruence of open intervals or point pairs. A formal, logical development of the theory of the congruence of point pairs is elaborated employing the logical machinery of the author's Introduction to mathematical logic and its applications, University of Miami Press, 1950. Axioms essentially equivalent to those for the congruence of segments in Hilbert's Foundations of geometry are employed. A further axiom, "If $z z \cong x y$, then $x=y$," is needed if theorems are to be freed from tacit restrictions and given added generality and precision. The definitions of the relation "less than" among open intervals and of the "sum" of two intervals employed, for example, by R. L. Moore (Trans. Amer. Math. Soc. (1908)) require modification, for the reason Hilbert's 
axioms require supplementation. The emended definitions are: $x y<z w: \rightleftarrows:[x=y . z$ $\neq w . v].(E p)(p e(z w) . z p \cong x y)$ on the one hand, and on the other: (a) $x \neq y: . \rightarrow:$. (z is in the extension of $x y$ beyond $y[$ or $z=y]$ and $y z \approx u v: \rightleftarrows: x y+u v=x z$, and (b) $[x=y$. $\rightarrow . x y+u v=u v+x y$.]. Novel are the bracketed portions of the above definitions. (Received January 10, 1951.)

\section{Statistics And Probability}

\section{6t. R. E. Fortet: Additive functionals of a Markoff process.}

Let $X(t)$ be a Markoff process of a continuous (for instance) parameter $t ; X$ takes its values in a space $\mathfrak{X}$, and $F(t, x ; \tau, e)(e$ : subset of $\mathfrak{X})$ is its transition probability. Let $L(t, \tau)$ be a random variable corresponding to each interval $(t, \tau)(\tau<t)$ with the following properties: (a) $L(t, \tau)=L\left(t, t^{\prime}\right)+L\left(t^{\prime}, \tau\right)\left(t<t^{\prime}<\tau\right)$; (b) Conditionally if $X(t)=x$, $X(\tau)=\xi, L(t, \tau)$ and $X\left(t^{\prime}\right)$ are independent if $t^{\prime}<t<\tau$ or $t<\tau<t^{\prime}$; (c) Conditionally when $X(t)=x, X\left(t^{\prime}\right)=x^{\prime}, X(\tau)=\xi\left(t<t^{\prime}<\tau\right), L\left(t, t^{\prime}\right)$ and $L\left(t^{\prime}, \tau\right)$ are independent. $L(t, \tau)$ will be called an additive functional of $X(t)$; if $\psi(t, x ; \tau, \xi ; v)$ is the characteristic function of $L(t, \tau)$, conditionally when $X(t)=x, X(\tau)=\xi(t<\tau)$, and if one puts: $\mu(t, x ; \tau, e ; v)=\int_{e} \psi(t, x ; \tau, \xi ; v) d \xi F(t, x ; \tau, e)$, one obtains the fundamental relation: $\mu(t, x ; \tau, e ; v)=\int_{\mathfrak{X}^{\mu}}\left(t^{\prime}, x^{\prime}, \tau, e ; v\right) d_{x^{\prime} \mu}\left(t, x ; t^{\prime}, e^{\prime}\right)\left(t<t^{\prime}<\tau\right)$. Assume that: $\lim _{\Delta t \rightarrow+0}$ $(\mu(t, x ; t+\Delta t, \mathfrak{X} ; v)-1) / \Delta t=\lim _{\Delta t \rightarrow+0}(\mu(t-\Delta t, x ; t, \mathfrak{X} ; v)-1) / \Delta t=A(t, x ; v)$ exists; under some conditions, one can prove that (1) $\mu(t, x ; \tau, e ; v)=F(t, x ; \tau, e)$ $+\int_{t}^{t} d u \int_{\mathfrak{X}}(u, y ; \tau, e ; v) A(u, y ; v) d_{y} F\left(t, x ; u, e^{\prime \prime}\right)$. If $X(t)$ is a real number, under some conditions it follows from (1) that $\partial \mu / \partial t+(\alpha / 2)\left(\partial^{2} \mu / \partial x^{2}\right)+\beta \partial \mu / \partial x+A \mu=0$, where $\alpha$ and $\beta$ are the coefficients of the Kolmogoroff equation of $X(t)$. (Received January 15, 1951.)

\section{7t. R. E. Fortet: Estimation of eigenvalues by Monte Carlo methods.}

Given an integral equation of the form (1) $f(t)=\lambda \int_{a}^{b} \Gamma(t, \tau) f(\tau) d \tau$, where $\Gamma(t, \tau)$ is a definite positive kernel, one obtains an estimation of the Fredholm determinant $D(\lambda)$ of (1) in the following way: let $X^{*}(t)$ be the Gaussian stochastic process with covariance equal to $\Gamma(t, \tau)$; the characteristic function $\phi(v)$ of the random variable $Y=\int_{a}^{b} X^{* 2}(t) d t$ is equal to $[D(2 i v)]^{-1 / 2}$; the difficulty is to "realize" $X^{*}(t)$; a procedure is suggested which replaces $X^{*}(t)$ by an approximate process of the form $X(t)$ $=\left(1 / m^{1 / 2}\right) \int_{-\infty}^{+\infty} R(t, \tau) d N^{*}(\tau)$ where $N^{*}(\tau)$ is a centered Poisson process of parameter $m ; m$ has to be sufficiently large; $R$ is any solution of $\Gamma(t, \tau)=\int_{-\infty}^{+\infty} R(t, u) R(\tau, u) d u$ $(a \leqq t, \tau \leqq b)$. If $\Gamma(t, \tau)$ depends on $(\tau-t)$ only, it is easy to find the solutions $R$ which depend on $(\tau-t)$ only; in the general case one can prove the existence of at least one solution under weak conditions. (Received January 15, 1951.)

\section{8t. R. E. Fortet: Random determinants.}

The following investigations are an attempt to solve problems arising in so-called "linear programming." Let $D_{n}$ be a determinant, of any order $n$, of the form: $D_{n}$ $=\left\|x_{i j}+X_{i j}\right\|$, where the $x_{i j}$ 's are given numbers, and the $X_{i j}$ 's random variables; one can compute $E\left(D_{n}\right)$ and $E\left(D_{n}^{2}\right)$, under some assumptions, for instance if the $X_{i j}$ 's are independent and if $E\left(X_{i j}\right)=0, E\left(X_{i j}^{2}\right)=\sigma_{i}^{2}<+\infty$. In a Euclidean $n$-dimensional space $E_{n}$, let $P_{1}, \cdots, P_{n}$ and $\phi$ be $(n+1)$ given points, and $M_{1}, \cdots, M_{n}$ be $n$ random 
points; let $P$ and $\Pi$ be the planes $\left(P_{1}, \cdots, P_{n}\right)$ and $\left(M_{1}, \cdots, M_{n}\right)$; let $h$ be the algebraic distance from $P$ to $\phi$; if $n \rightarrow+\infty$, and if $h$ remains $>0$ and constant, the probability that $\phi$ is above $\Pi$ tends toward 1 , under some assumptions [for instance, if the $\left(P_{i} M_{i}\right)^{\rightarrow}$ are independent Laplacian vectors with $E\left(\left(P_{i} M_{i}\right)^{\rightarrow 2}\right)$ equal to a constant smaller than $1 / 2$, and if $P_{i} P_{j}=1$ for any $\left.i, j, n\right]$; the preceding results on $D_{n}$ can be applied to a more precise study of the distance from $I I$ to $\phi$. (Received January 15,1951 .)

\section{9t. R. E. Fortet: On some functionals of Laplacian processes.}

Let $N^{*}(t)$ be a centered Poisson process, with parameter $m$; and $R(t, \tau)$ a (not random) function such that $\int_{-\infty}^{+\infty} R(t, \tau)^{2} d \tau<+\infty$ for any given $t$. The process $X(t)$ defined by: $X(t)=\lim$ m.q. $\alpha \rightarrow-\infty, \beta \rightarrow+\infty$ i.a.c. $\left(1 / m^{1 / 2}\right) \int_{\alpha}^{\beta} R(t, \tau) d N^{*}(\tau)$ is called a random function derived from a Poisson process (f.d.P.). Assume that $R(t, \tau)=R(\tau-t)$ depends on $(\tau-t)$ only; and let $V(x)$ be a function such that (1) $\left|V(x)-V\left(x^{\prime}\right)\right|$ $\leqq M\left|x-x^{\prime}\right|^{\alpha}(0<\alpha \leqq 1 ; M$ a constant depending on $V)$. We put $Y(t)=V[X(t)]$, $L(t, \tau)=\int_{t}^{\tau} Y(u) d u$. If $\int_{-\infty}^{+\infty}\left[\int_{|u|>a} R^{2}(u) d u\right]^{\alpha / 2} d a<+\infty$ and, except in an exceptional case, for any fixed $t$, the distribution function of $L(t, t+T)$, when properly normed, tends toward the Laplace law if $T \rightarrow+\infty$. Other results of the same kind can be obtained. These results are extended to the case where $X(t)$ is a Laplacian stationary process instead of an f.d.P., and to cases where $X(t)$ (f.d.P. or Laplacian process) is not stationary. Assumption (1) can be weakened. (Received January 12,1951.)

\section{TOPOLOGX}

\section{W. R. Baum: The group of reduced null-spherods.}

Let $\Psi^{n}(K)$ be the group of $n$-dimensional null-spherods of a complex $K, \Psi^{n}(K)$ $\cong \Gamma^{n}\left(K^{n}\right) / \mathrm{P}_{0}^{n}\left(K, K^{n}\right)$. (Cf. W. R. Baum, Bull. Amer. Math. Soc. Abstract 57-2-162.) If $\Pi_{0}^{n}\left(K^{n}\right)$ is the subgroup of the Hurewicz homotopy group $\pi_{n}\left(K^{n}\right)$ generated by all the differences $\alpha-\alpha^{\prime}$, where the elements $\alpha, \alpha^{\prime} \in \pi_{n}\left(K^{n}\right)$ are free-homotopic on the $n$-dimensional skeleton $K^{n}$ of $K$ (cf. H. Hopf, Comment. Math. Helv. vol. 17 (1945) pp. 307-326), consider the subgroup $\Psi_{0}^{n}(K)$ of $\Psi^{n}(K)$ which corresponds to $\Pi_{0}^{n}\left(K^{n}\right) / \mathrm{P}_{0}^{n}\left(K, K^{n}\right)$. For $n=1, \Psi_{0}^{n}(K)=\Psi^{n}(K)$. The factor-group $\Omega^{n} \cong \Psi^{n} / \Psi_{0}^{n}$ depends only on the skeleton $K^{n}$, but is a topological invariant of $K$. The group $\Omega^{n}$ can be considered as the group of the "reduced" $n$-null-spherods (in a certain geometric sense). If $K$ is aspherical in the dimensions $r(1<r<n)$ it follows from a theorem due to H. Hopf (loc. cit., Theorem I) that the structure of $\Omega^{n}(n>1)$ is determined by the structure of the fundamental group of $K$. (Received January 10,1951.)

\section{P. V. Reichelderfer: On the barycentric homomorphism in a singular complex.}

Rad6 has introduced a singular complex $R$ in which no a priori identifications are made (T. Rad6, On identifications in singular homology theory, to appear in Rivista di Matematica della Universitá di Parma), and has devised the following technique for studying the effect of certain a priori identifications on the theory (T. Rad6, $A n$ approach to singular homology theory, unpublished). A system of subgroups $G_{p}$ of the chain groups $C_{p}$ is termed an identifier if the relation $\partial G_{p} \subset G_{p-1}$ is satisfied, so that the $G_{p}$ form a subcomplex $G$ of $R$. The identifier is said to be unessential in $R$ if the 
natural homomorphism $\pi: R \rightarrow R / G$ induces an isomorphism onto between the corresponding homology groups. The main purpose of this note is to show that the nuclei of the barycentric homomorphisms constitute an unessential identifier in $R$, and to improve certain results obtained by Rado. In order to establish these results, explicit formulas for the barycentric homomorphisms and for a modified barycentric homotopy operator are developed. (Received December 18, 1950.)

\section{J. W. T. Youngs,} Associate Secretary 